\title{
LABORATORIO A DISTANCIA PARA LA PRUEBA Y EVALUACIÓN DE CONTROLADORES A TRAVÉS DE INTERNET
}

\author{
Aldo R. Sartorius C. \\ Universidad Central "Marta \\ Abreu” de las Villas, \\ Departamento de Automática y \\ Sistemas Computacionales, \\ Carretera a Camajuaní Km. 51/2 \\ Santa Clara, Villa Clara, Cuba \\ sartorius@uclv.edu.cu
}

\author{
Luis Hernández S. \\ Universidad Central "Marta \\ Abreu” de las Villas, \\ Departamento de Automática y \\ Sistemas Computacionales, \\ Carretera a Camajuaní Km. 51/2 \\ Santa Clara, Villa Clara, Cuba \\ luishs@uclv.edu.cu
}

\author{
Rafael Aracil Santonja. \\ Universidad Politécnica de \\ Madrid, \\ División de Ingeniería de \\ Sistemas y Automática, \\ José Gutiérrez Abascal 2 E- \\ 28006 Madrid, España
}

\section{RESUMEN}

En este trabajo es presentado el Sistema de Laboratorios a Distancia (SLD), el cual es un laboratorio a distancia enfocado al estudio del control automático desarrollado en la Universidad Central "Marta Abreu" de las Villas. El principal objetivo de este laboratorio es permitir a los usuarios aprender a ajustar controladores predefinidos y a diseñar sus propios controladores para posteriormente probarlos sobre un conjunto de dispositivos físicos a través de Internet y analizar su desempeño. El SLD utiliza herramientas de diseño de sistemas de control asistido por computadora como Matlab-Simulink para la creación de nuevos controladores, los cuales pueden hacer uso de SFunctions desarrolladas utilizando el lenguaje C, lo que posibilita la creación de controladores complejos de una forma sencilla. El SLD permite una rápida y fácil integración de nuevos procesos para realizar pruebas de controladores y en la actualidad están disponibles tres dispositivos: un motor de CD y un robot manipulador en Cuba así como un cilindro electro neumático en España. El SLD ha sido utilizado durante el último año en cursos de pregrado en las materias de identificación y teoría de control en las Universidades "Marta Abreu” de las Villas y

Artigo Submetido em 13/04/04

1a. Revisão em 05/07/04;

Aceito sob recomendação do Ed. Assoc. Prof. José R. C. Piqueira en la Universidad de Cienfuegos en Cuba, así como en cursos de postgrado impartidos en México en las materias de teoría avanzada de control y control de robots manipuladores.

PALABRAS CLAVE: Controladores, Laboratorio a distancia, S-Functions.

\section{INTRODUCCIÓN}

Debido principalmente al desarrollo y uso de Internet y de las tecnologías Web en los últimos años la educación ha presentado un cambio radical. La enseñanza basada en Web, los cursos a distancia, los libros electrónicos y las plataformas interactivas de educación a distancia juegan un papel cada vez más importante en el proceso de aprendizaje. En (Alhalabi et al, 1998) el autor analiza el estado del arte de la educación a distancia. Un amplio estudio sobre los sistemas educacionales basados en Web es proporcionado en (Ausserhofer, 1999) donde se realiza una descripción detallada de los problemas actuales concernientes al diseño de sistemas educacionales basados en Web y sus posibles soluciones. En (Shor, 2000) el autor proporciona algunas recomendaciones en cuanto a las características que deben tener los laboratorios a distancia, además de analizar los beneficios económicos y de cooperación que representa su uso. El área de control automático es una de las áreas técnicas que más ha explotado las nuevas tecnologías para desarrollar herramientas que faciliten el aprendizaje a distancia (Casini 
et al, 2003). Un profundo análisis sobre la enseñanza del control automático usando las nuevas tecnologías Web es reportado en (Sebastián, 2002). En (Poindexter y Heck, 1999) se presenta una guía para utilizar los sistemas de educación a distancia basados en Web en el área del control automático, prestando especial atención al uso de laboratorios a distancia.

Los laboratorios a distancia son laboratorios donde los usuarios pueden interactuar con dispositivos reales a través de Internet. Normalmente los usuarios a través de una interfaz Web pueden cambiar algunos parámetros de control, realizar experimentos, ver los resultados y descargar los datos del experimento. Este tipo de experimentos son utilizados por ejemplo en (Gillet et al, 1997), (Puerto et al, 2001) y en (Leiner, 2002), donde se presentan laboratorios para controlar a distancia motores de corriente directa; en (Messom y Craig, 2002) es descrito un laboratorio para controlar un robot móvil y en (Henry, 1999) están disponibles varios experimentos de control de procesos de forma remota. Conforme las tecnologías Web fueron presentado mas alternativas, los laboratorios a distancia fueron evolucionando principalmente en la forma de realimentar información al usuario y en el uso de dispositivos cada vez más complejos para controlar como por ejemplo el sistema presentado en (Calkin et al, 1998), donde se presenta un laboratorio para controlar un robot de seis grados de libertad. En (Bauchspiess et al, 2003) y en (Ko et al, 2001) son presentados varios experimentos para controlar nivel en tanques interconectados mientras que en (Dixon et al, 2001) están disponibles varios experimentos con sistemas mecánicos.

Esta evolución hacia dispositivos más complejos ha requerido también la implementación de controladores más complejos para garantizar los objetivos de control, como por ejemplo los presentados en (Hahn y Spong, 2000) donde se presentan varios tipos de controladores PID así como controladores por dinámica inversa para controlar distintos dispositivos mecánicos o los presentados en (Jing Zhang et al, 2001) donde se ofrecen controladores en el espacio de estados y controladores fuzzy para controlar un helicóptero con dos grados de libertad.

En los últimos años la tendencia en el desarrollo de laboratorios a distancia es permitir que los usuarios desarrollen sus propios controladores de forma remota, en estos laboratorios la complejidad en el diseño del hardware y principalmente del software se incrementa drásticamente. Un ejemplo de esto es el caso del sistema presentado en (Piguet y Guillet, 1999), donde los usuarios pueden desarrollar controladores utilizando scripts en el lenguaje de Matlab para controlar un péndulo invertido. En el laboratorio propuesto en (Chiculita y Frangu, 2002) los usuarios pueden desarrollar sus propios controladores utilizando el lenguaje Phyton; este código es mandado al servidor del laboratorio a distancia en donde es compilado e implementado para llevar a cabo experimentos de control de nivel en tanques. Otro enfoque es presentado en (Chang y Hung, 2000), donde los usuarios pueden implementar sus propios controladores de forma remota utilizando una API escrita en lenguaje C. Este código es compilado por el servidor del laboratorio remoto y cargado en una tarjeta provista con un procesador digital de señales (DSP) que se encarga de controlar un motor con un péndulo invertido. En (Bonivento et al, 2002) es presentado un laboratorio a distancia que permite la creación de controladores de forma remota utilizando el ambiente Matlab-Simulink para realizar varios experimentos de control de procesos, mientras que en (Casini et al, 2003) se utiliza el mismo enfoque pero para controlar dispositivos mecánicos de forma remota.

Este artículo describe el diseño y construcción de un laboratorio a distancia enfocado al estudio del control automático que ha sido desarrollado en la Universidad Central "Marta Abreu" de las Villas. El Sistema de Laboratorios a Distancia (SLD) permite a los usuarios cambiar las referencias, modificar los parámetros de control, escoger el tiempo de muestreo y diseñar sus propios controladores de una forma sencilla utilizando herramientas ampliamente conocidas en el área del control automático como son Matlab y Simulink. Una de las principales características del SLD es que los usuarios remotos pueden crear controladores que no estén restringidos solo a los bloques provistos por Simulink, ya que permite el desarrollo de controladores que utilicen SFunctions definidas por el usuario en lenguaje C; esto hace posible la prueba y evaluación de controladores complejos de una forma sencilla.

Este artículo es organizado de la siguiente forma: La sección 2 describe las características comunes y especificas del SLD así como algunas notas sobre su funcionamiento general. La sección 3 describe el uso del SLD desde un punto de vista práctico. La sección 4 explica la creación de controladores por parte de los usuarios. En la sección 5 son comentadas algunas experiencias del uso del SLD mientras que las conclusiones son mostradas en la sección 6 .

\section{CARACTERÍSTICAS DEL SISTEMA}

El SLD es un laboratorio a distancia cuyo principal objetivo es permitir a los usuarios aprender a ajustar controladores predefinidos y a diseñar sus propios controladores para probarlos en dispositivos reales a través de Internet.

\subsection{Características comunes de los laboratorios virtuales y remotos}

Al igual que otros laboratorios a distancia (Bauchspiess et al, 2003)(García et al, 2003), el SLD posee algunas 
características que son comunes con la gran mayoría de los laboratorios a distancia que operan actualmente tales como:

Disponibilidad: Los sistemas de enseñanza basados en Web deben de poder estar disponibles las 24 horas del día. Esto implica que el sistema debe de tener medidas de autoprotección para garantizar este aspecto. Todos los experimentos deben de ser equipados con dispositivos hardware y software que prevengan daños al equipo o al personal presente en el laboratorio.

Accesibilidad: Debido a que el SLD esta montado sobre una plataforma Web, permite a los usuarios acceder al sistema desde cualquier parte del mundo. Para ello solo es necesaria una computadora con conexión a Internet y un navegador Web, tales como el Internet Explorer o Netscape Navigator.

Facilidad de uso: Para usar el sistema los usuarios solo deben tener los conocimientos básicos de los sistemas de control, tales como el modelado de sistemas y el diseño de controladores. De esta forma el usuario se centra en aprender estos temas y evita todos los problemas asociados a la implementación y operación de los equipos usados en las prácticas.

\subsection{Características especificas del SLD}

Además de las características descritas anteriormente, las cuales son comunes a la mayoría de los laboratorios a distancia, el SLD posee características adicionales que son comentadas a continuación:

Interfaz de usuario rápida y fácil: Una parte muy importante en el desarrollo de un sistema de enseñanza basado en Web es la interfaz de usuario. La principal función de esta parte del sistema es conformar el pedido de las prácticas y mandarlo hacia el servidor Web. La interfaz de usuario del SLD esta basada en páginas HTML que utilizan funciones Javascript y ASP; esto permite que los usuarios puedan acceder al sistema de una forma rápida y sin necesidad de descargar o instalar ningún software adicional. El sistema cuenta también con páginas de ayuda que proporcionan información técnica a los usuarios, tal como el modelado matemático de los dispositivos usados en las prácticas, datos del fabricante y ajuste de reguladores entre otros tópicos de interés para las prácticas.

Administración de múltiples pedidos en forma paralela: El SLD permite atender múltiples pedidos de forma paralela administrando de forma centralizada dispositivos similares que se encuentren geográficamente separados pero unidos por redes de área extensa (WAN). Esto permite una mayor disponibilidad de los equipos y un servicio más rápido y eficiente para los usuarios; lográndose con esto reducir los tiempos de espera para que un usuario remoto realice una determinada práctica.
Desarrollo de controladores de forma remota usando Matlab y Simulink: Una de las características más importantes del SLD es que permite a los usuarios diseñar sus propios controladores utilizando el ambiente MatlabSimulink. Estos programas son un estándar en el área del control automático, por lo que los usuarios no necesitan perder tiempo aprendiendo nuevos lenguajes de programación para implementar un nuevo controlador, solo necesitan un conocimiento básico del ambiente de Matlab y Simulink. A través de la interfaz grafica de Simulink, una gran cantidad de bloques pueden ser escogidos $y$ conectados de forma muy sencilla, lo que permite a los usuarios crear controladores analógicos, digitales o híbridos de una forma muy rápida. Adicionalmente el SLD no restringe a los usuarios a usar solo los bloques provistos por Simulink, sino que permite de forma opcional, la creación de controladores complejos que incluyan S-Functions definidas por el usuario. Estas S-Functions deben ser implementadas utilizando el lenguaje de programación C, el cual es un lenguaje muy potente y ampliamente conocido por la mayoría de los estudiantes de ingeniería en la actualidad.

Tipo de controlador: Todos los experimentos en el SLD pueden ser controlados de dos formas distintas: con controlador predefinido o con controlador definido por el usuario. En el primer caso los usuarios deben asignar los valores a las distintas ganancias del controlador, por ejemplo el usuario puede escoger un controlador PID y cambiar los parámetros de las ganancias proporcional, integral y derivativa para ver la respuesta del sistema. En el segundo caso los usuarios pueden diseñar su propio controlador (como se ha explicado en el punto anterior) y mandarlo al SLD donde se implementara y será usado para controlar el experimento.

Cambio del periodo de muestreo: El SLD permite a los usuarios variar el periodo de muestreo de los experimentos. Esto permite probar como afecta este parámetro en el diseño de controladores digitales y en general en el desempeño de cualquier experimento.

Cambio de referencias: El sistema permite cambiar las referencias de los experimentos para poder comprobar el desempeño de un determinado controlador ante distintas señales de entrada.

\subsection{Funcionamiento general del SLD}

El SLD esta dividido en tres partes:

1. Interfaz del usuario.

2. Administración de los pedidos de las prácticas.

3. Procesamiento de las prácticas. 
La figura 1 muestra la interacción de estas tres partes. Los usuarios interactúan con el sistema a través de Internet. Al acceder al sitio Web del sistema se elige la práctica que se desea realizar. Allí el usuario debe llenar correctamente todos los datos en el formulario asociado a la práctica y finalmente elegir entre ejecutarla de manera simulada o real. El CGI que se encuentra en el servidor Web recibe los datos y los manda al Servidor de Administración de Prácticas (SAP) como un nuevo pedido. El SAP verifica cual estación de trabajo puede realizar la práctica pedida y una vez que la encuentra saca el pedido de la lista y se lo envía al Cliente de Administración de Prácticas (CAP) instalado en la estación de trabajo. Cuando el pedido llega al CAP se procesan los datos y se lleva a cabo la práctica utilizando Matlab-Simulink. Una vez que la práctica ha sido procesada se trasmite el resultado en sentido inverso al que trajo el pedido para que al final llegue hasta el usuario la respuesta de la práctica. La respuesta es una página Web que muestra los resultados del procesamiento.

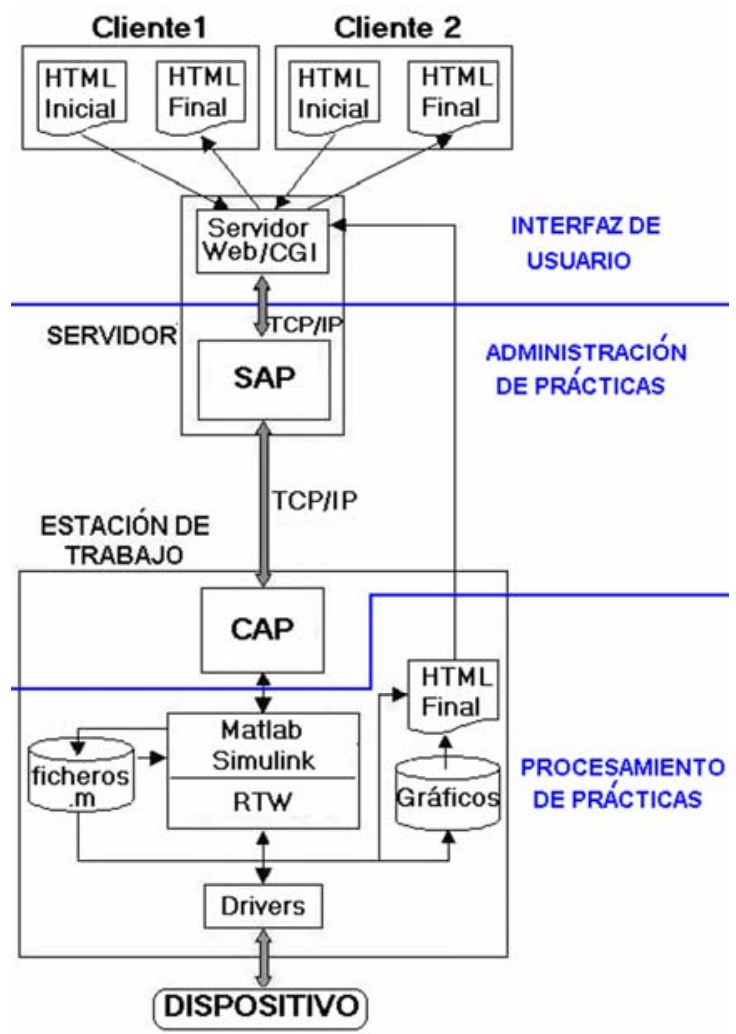

Figura 1. Funcionamiento general del Sistema de Laboratorios a Distancia (SLD).

El servidor Web y el Servidor de Administración de Prácticas $(S A P)$ se encuentran instalados en una computadora que utiliza Windows 2000 como sistema operativo. Las estaciones de trabajo necesitan tener instalado el Cliente de Administración de Prácticas (CAP) así como Matlab y Simulink para el procesamiento de las prácticas. Las estaciones de trabajo utilizan Windows 98 como sistema operativo.

El sistema cuenta actualmente con tres procesos para prácticas de control: un motor de $\mathrm{CD}$, un robot manipulador Asea IRB-6 y un cilindro electroneumático. El motor de $\mathrm{CD}$ es un proceso de una sola entrada y una sola salida (SISO) muy simple que nos permite realizar experimentos de identificación, control de posición y control de velocidad sin disturbios de carga, el cilindro electroneumático es también un proceso SISO pero que exhibe un comportamiento no lineal, en el se pueden realizar prácticas de control de posición del vástago, por ultimo el robot manipulador es un sistema de múltiples entradas y múltiples salidas (MIMO) que presenta una dinámica altamente acoplada y fuertemente no lineal, lo que nos permite realizar prácticas mas complejas de control de posición y seguimiento de trayectorias.

\section{USO DEL SISTEMA DE LABORATORIOS A DISTANCIA}

Cuando los usuarios acceden al sitio Web del SLD lo primero que tienen que hacer es registrarse en el sistema proporcionando su nombre de usuario y su clave. Esto permite tener dos tipos de usuarios: usuarios de prácticas y administradores. Una vez dentro del sistema los usuarios de prácticas pueden llevar a cabo varias operaciones, tales como ver las páginas de teoría, contactar con los desarrolladores del sistema así como ver las prácticas disponibles en el sistema. Todas estas páginas se encuentran localizadas en el servidor Web del sistema, el cual es común para todas las prácticas. Después de que el usuario a seleccionado alguna práctica, aparece una página Web que contiene el diagrama de bloques del sistema, una explicación de la simbología utilizada en la práctica, enlaces a las páginas de teoría de la práctica y un formulario en donde el usuario puede modificar los parámetros del controlador, el tiempo de muestreo, el valor o tipo de referencia o crear un controlador definido por el usuario.

\subsection{Prácticas con controlador predefinido}

En este tipo de prácticas el usuario sólo necesita un navegador de Web (Netscape, Internet Explorer u otro) para acceder al sitio Web del SLD. Una página de este tipo aparece en la figura 2, donde se muestra una práctica para evaluar el desempeño de un controlador PID desacoplado en un robot manipulador con dos grados de libertad. Adicionalmente se puede optar por dos formas de ejecución de la práctica: de forma simulada, donde se simula la ejecución y se obtiene una respuesta idealizada de la práctica o de forma real. 


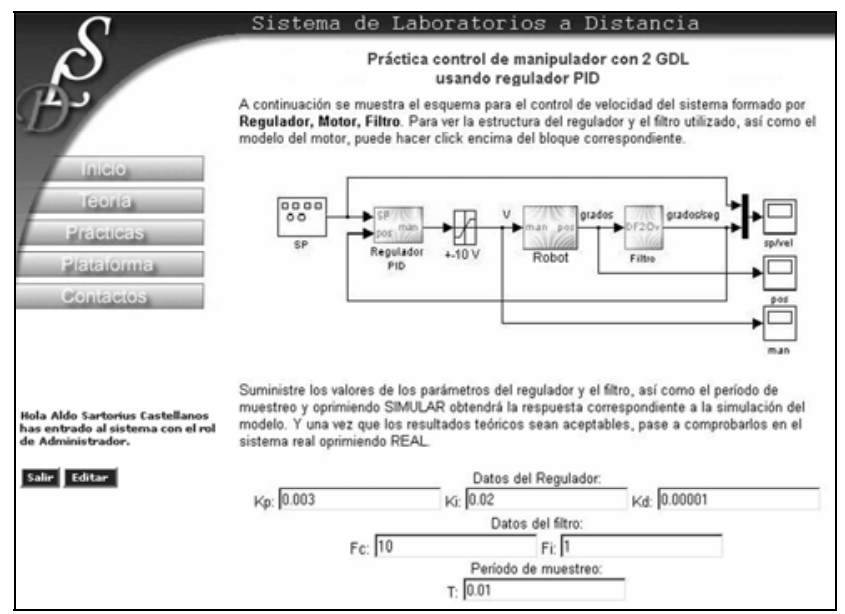

Figura 2. Página Web para práctica con controlador PID predefinido.

\subsection{Prácticas con controlador definido por el usuario}

Cuando el usuario accede a alguna práctica en la que se puede definir el controlador a usar, aparece una página Web como la mostrada en la figura 3. De ella el usuario debe descargar un archivo de Simulink que contiene el diagrama de bloques de la práctica a realizar. Para llevar a cabo este tipo de prácticas el usuario necesita tener instalado el software Matlab-Simulink para poder modificar el archivo de Simulink descargado, tal como se explicara en la sección siguiente.

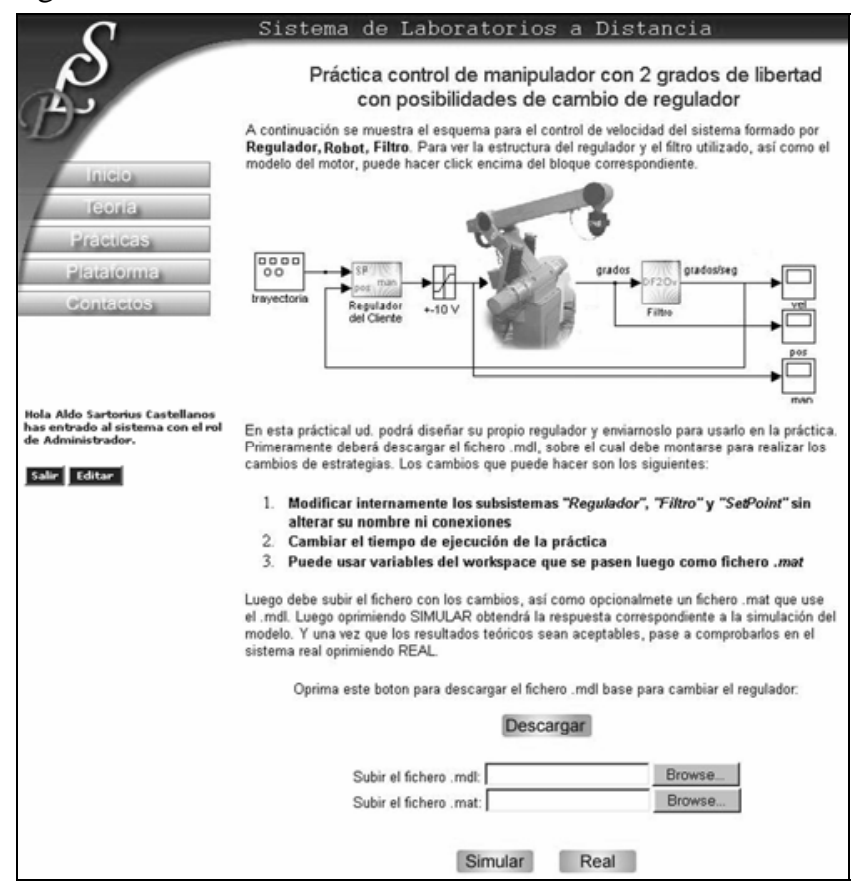

Figura 3 Página Web para práctica con controlador definido por el usuario.

\section{CREACIÓN DE CONTROLADORES}

Una de las características más importantes del SLD es que permite a los usuarios la creación de sus propios controladores de forma remota. Estos controladores pueden ser creados utilizando solo bloques de Simulink o, de forma opcional, una combinación de bloques de Simulink con funciones definidas por el usuario. Para un mejor entendimiento se presentan ejemplos de creación de controladores definidos por el usuario para los dos casos: primero en un motor de corriente directa y posteriormente para un robot manipulador Asea IRB-6.

\subsection{Creación de controladores utilizando bloques de Simulink (motor de CD)}

Cuando el usuario selecciona una práctica con posibilidad de crear un controlador, se le muestra una página Web que contiene un formulario como el mostrado en la figura 4. En esta página el usuario debe descargar un archivo que contiene el diagrama de bloques de la práctica (Motor.mdl es este caso). En este archivo el usuario debe modificar los subsistemas Referencia y Controlador utilizando el software Matlab-Simulink, sin alterar el nombre de las entradas y salidas de los subsistemas tal como se muestra en la figura 5, en donde el usuario utiliza una suma de tres señales senosoidales como referencia en el subsistema Referencia y ha creado un controlador PID dentro del subsistema Controlador.

\begin{tabular}{|c|c|}
\hline \multicolumn{2}{|l|}{ Descargar } \\
\hline Subir fichero. $\mathrm{mdl}$ Motor.mdl & Examinar \\
\hline Subir fichero mat & Examinar... \\
\hline \multicolumn{2}{|l|}{${ }^{\star}$ Opcional } \\
\hline \multirow[t]{2}{*}{$\ulcorner$ Crear S-Function } & \\
\hline & \\
\hline
\end{tabular}

Figura 4. Formulario para práctica con controlador definido por el usuario usando Simulink.

Una vez realizada la modificación, el usuario debe subir al servidor el fichero modificado y decidir si quiere realizar una simulación o controlar el proceso real. Cuando se ha mandando ha ejecutar el proceso real el SLD realiza primero una simulación del sistema y sobre los datos obtenidos de la misma se realizan una serie de pruebas para determinar si el controlador se puede implementar en el sistema real. En el caso del motor de CD estas pruebas se centran en determinar si el sistema presenta oscilaciones de alta frecuencia que puedan dañar al motor mecánicamente o por temperatura. Una vez determinados estos aspectos el SLD se encarga de implementar el controlador creado, compilar el sistema y realizar la práctica en tiempo real. 


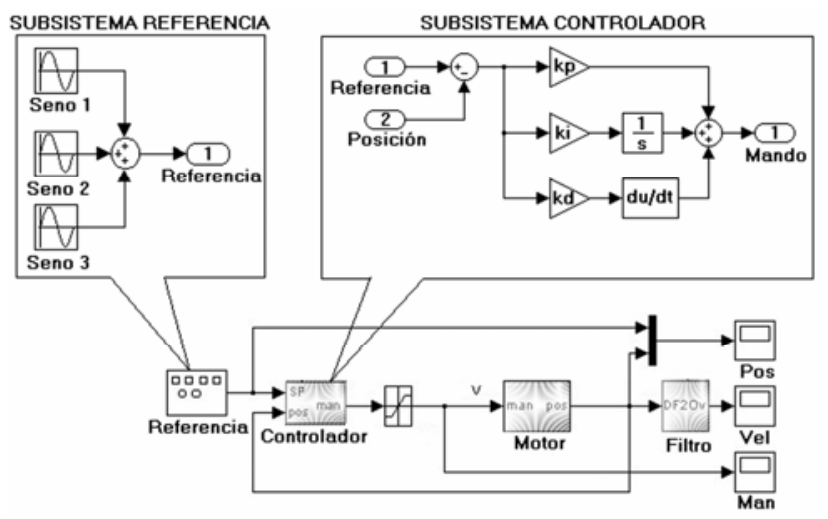

Figura 5. Diagrama de bloques con referencia y regulador PID implementado por el usuario.

En caso de que el SLD detecte que el controlador desarrollado representa un riesgo para el motor, se le informa al usuario la causa y se le proporcionan los datos y graficas de la simulación realizada para que pueda examinar el comportamiento del sistema.

\subsection{Creación de controladores utilizando bloques de Simulink y S-Fuctions definidas por el usuario (robot manipulador)}

A través de este mecanismo el SLD permite la creación de controladores complejos, los cuales no esta restringidos al uso de bloques de Simulink, si no que permite a los usuarios crear sus propios bloques a través del uso de SFunctions escritas en lenguaje C. Cuando el usuario selecciona una práctica con posibilidad de crear un controlador y activa la casilla de creación de S-Function, se le muestra una página Web que contiene un formulario similar al del ejemplo anterior (figura 4) pero que contiene cinco campos adicionales, como el mostrado en la figura 6.

Los campos adicionales son comentados a continuación:

Nombre: Aquí se debe especificar el nombre de la SFunction. Este será el nombre del bloque definido por el usuario.

Número de entradas: Especifica el número de entradas multiplexadas que entraran al bloque definido por el usuario.

Número de salidas: Especifica el número de salidas demultiplexadas que saldrán del bloque definido por el usuario.

Función principal: Aquí el usuario debe escribir el código de la S-Function especificando las entradas al bloque definido por el usuario como u[0], u[1]...u[n] y las salidas como $\mathrm{y}[0], \mathrm{y}[1] \ldots \mathrm{y}[\mathrm{n}]$. Este código debe ser escrito en lenguaje $\mathrm{C}$.

Funciones auxiliares: Aquí el usuario puede escribir la declaración de las funciones auxiliares, las cuales serán llamadas desde la función principal. El código debe ser escrito en lenguaje $C$.

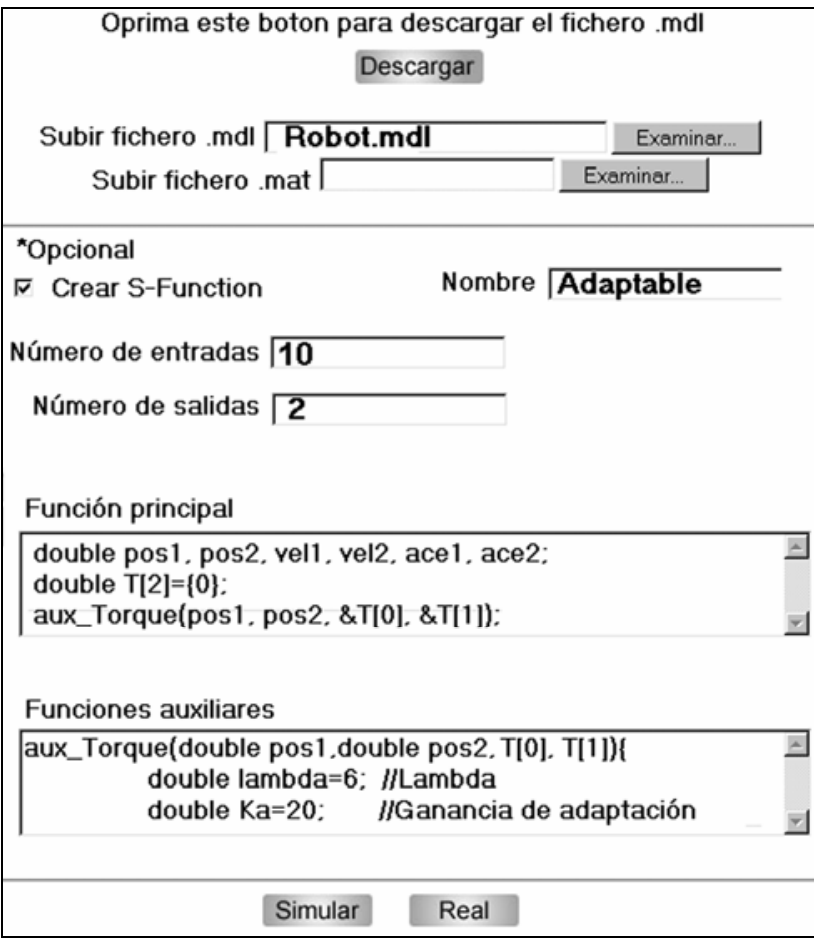

Figura 6. Formulario de práctica con controlador definido por el usuario usando Simulink y SFunction.

Al igual que en el ejemplo anterior, en esta página Web el usuario debe descargar un archivo que contiene el diagrama de bloques de la práctica (Robot.mdl en este caso). En este archivo el usuario debe modificar el subsistema Controlador utilizando el software Matlab-Simulink sin alterar el nombre de las entradas y salidas del subsistema tal como se muestra en la figura 7, donde el usuario a implementado un controlador PD con compensación adaptable propuesto en (Slotine y W., 1991). Este controlador utiliza una función definida por el usuario a través de una S-Function llamada "Adaptable" en el subsistema controlador. En los cinco campos adicionales del formulario el usuario deberá poner el nombre de la SFunction (en este caso Adaptable), el número de entradas (10 entradas), el número de salidas (dos salidas) y escribir el código principal de la función así como las funciones auxiliares que utilizara, tal como se muestra en la figura 6 . Una vez realizada la modificación y especificada la SFunction, el usuario debe decidir si quiere realizar una simulación o controlar el proceso real. Cuando se ha 
mandando ha ejecutar el proceso real, el SLD realiza primero una simulación del sistema así como una serie de pruebas, que en el caso del robot manipulador están centradas en tres aspectos:

1. Que la trayectoria cartesiana no sobrepase el espacio de trabajo del robot manipulador.

2. Que las posiciones articulares no sobrepasen los límites mecánicos de cada articulación.

3. Que el sistema no presente oscilaciones sostenidas de alta frecuencia que puedan desajustar mecánicamente al robot y/o dañar los actuadores del mismo.

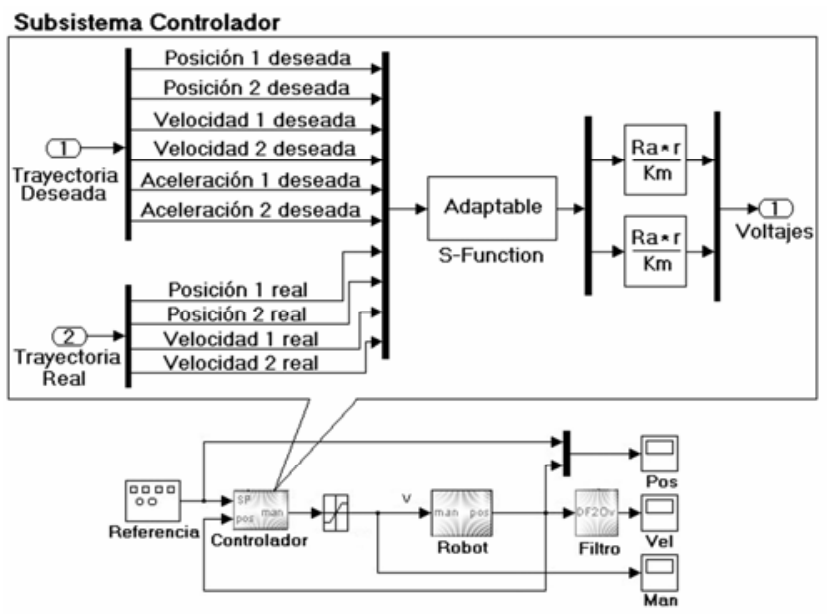

Figura 7. Controlador PD con compensación adaptable definido por el usuario utilizando bloques de Simulink y SFunction.

Una vez determinados estos aspectos el SLD se encarga de implementar el controlador, compilar el sistema y realizar la práctica en tiempo real. En caso de que el SLD detecte que el controlador desarrollado representa un riesgo para el robot manipulador, se le informa al usuario la causa y se le proporcionan los datos y graficas de la simulación realizada para que pueda examinar el comportamiento del sistema.

\section{EXPERIENCIAS DE USO DEL SLD}

Durante el año 2003 se realizaron las primeras pruebas del SLD desde México y España, con el fin de implementar controladores en los dispositivos localizados en Cuba. El SLD se utilizo para impartir cursos de postgrado en las materias de teoría avanzada de control y robótica en el Instituto Tecnológico de Minatitlán en México. Durante estos cursos el sistema trabajó de forma satisfactoria pero presentaba tiempos de espera grandes entre el momento de mandar el controlador y el momento de recibir la respuesta, por lo que se empezó a trabajar en dos vías:
1. Modificar el formato de las imágenes para tener una carga más rápida, así como revisar los algoritmos encargados de implementar los controladores, de forma que hubiera una reducción en el tiempo de procesamiento.

2. Realimentar al usuario los datos del proceso controlado, para que durante la espera de la página de respuesta pueda visualizar en un applet Java el estado del proceso durante el control. Esto permitirá que el usuario sepa que sé esta procesando la información y no piense que el sistema esta “colgado".

Las modificaciones referentes al primer punto se encuentran ya terminadas, mientras que las del segundo punto se encuentran actualmente en fase de desarrollo.

A partir del presente año el SLD ha sido utilizado en diferentes prácticas en cursos de identificación y control de accionamientos para las carreras de Ingeniería Mecánica e Ingeniería en Automática en la Universidad Central "Marta Abreu" de las Villas y en la Universidad de Cienfuegos, ambas localizadas en Cuba. Durante estas lecciones los usuarios mostraron un gran interés por controlar dispositivos de forma remota a través de Internet. Tras una breve explicación sobre el uso del sistema, los usuarios se registraron en el mismo y comenzaron a realizar experimentos en menos de cinco minutos. Uno de los aspectos que más estimulo a los estudiantes fue poder evaluar las diferencias entre los modelos matemáticos y las plantas reales. En promedio, con quince alumnos por grupo, se realizan entre 60 y 80 prácticas en menos de 30 minutos lo que da una idea de la alta tasa de aprovechamiento de los equipos al accederlos de forma remota utilizando Internet.

\section{CONCLUSIONES}

El Sistema de Laboratorios a Distancia (SLD) proporciona a los usuarios la facilidad de uso de paquetes como Matlab y Simulink en conjunto con el Toolbox Real Time Workshop (RTW) para la creación y prueba de controladores de forma remota en dispositivos reales.

La creación de un software de administración de prácticas que mantiene la filosofía y funciones del Matlab Web Server, pero que incorpora nuevas características que permiten, entre otras cosas, el intercambio de ficheros con los usuarios hace posible la creación de controladores de forma remota. Este mecanismo permite la creación de controladores que utilicen algoritmos complejos, los cuales pueden ser implementados de una forma sencilla utilizando la potencia y flexibilidad que provee el lenguaje C.

Como el software del sistema es fácilmente adaptable a nuevos procesos, la incorporación de nuevos dispositivos para experimentación de controladores se puede realizar de una manera sencilla. Debido a que el sistema esta aun en fase de desarrollo, el numero de dispositivos disponibles es 
limitado (un motor de corriente directa, un robot manipulador y un cilindro electroneumático), pero se esta trabajando para en un futuro incorporar nuevos procesos que incluyan experimentos para el control de temperatura, el control de nivel, entre otros procesos.

Asimismo la incorporación en un futuro de una mayor y mejor realimentación al usuario a través de la tecnología Java, hará al sistema más interactivo y estimulante para los usuarios.

\section{REFERENCIAS}

Alhalabi, B.; Hamza, M.K.; Hsu, S. y Romance, N. (1998). Virtual Labs vs. Remote Labs: Between Myth \& Reality. 7th Florida Higher Education Consortium, Deerfield Beach, Florida.

Ausserhofer, A., 1999, "Web-based teaching and learning: A panacea?". IEEE Magazine pp. 92-96.

Bauchspiess, A.; Guimaraes, B. y Gosmann, H.L. (2003). Remote Experimentation on a Three Coupled Water Reservoirs. IEEE International Symposium on Industrial Electronic, Rio de Janeiro, Brasil.

Bonivento, C.; Gentili, L.; Marconi, L. y Rappini, L. (2002). A Web Based Laboratory for Control Engineering Education. Second International Workshop on Teleeducation in Engineering Using Virtual Laboratories, Sherbrooke, Canada.

Calkin, D.W.; Parkin, R.M.; Safaric, R. y Czarnecki, C.A. (1998). Visualization, Simulation \& Control of A Robotic System Using Internet Technology. 5th IEEE International Workshop on Advanced Motion Control, Coimbra Portugal.

Casini, M.; Prattichizzo, D. y Vicino, A. (2003). ELearning by Remote Laboratories: A New Tool for Control Education. 6th IFAC Symposium on Advances in Control Education, Oulu, Finlandia.

Chang, T. y Hung, D. (2000). Web Based Distance Experiments: Design and Implementation. International Conference on Engineering Education, Taipei, Taiwan

Chiculita, C. y Frangu, L. (2002). A Web Based Remote Control Laboratory. The 6th Word Multiconference on Systemics, Cybernetics and Informatics, Orlando Florida.

Dixon, W.E.; Dawson, D.M. y Costic, B.T. (2001). Towards the Standardization of a MATLAB-Based Control System Laboratory Experience for
Undergraduate Students. American Control Conference, Arlington VA.

García, R.M.; Wornie, F.; Stewart, B.G. y Harrison, D.K. (2003). Real-Time Remote Network Control of an Inverted Pendulum Using ST-RTL. 32nd ASEE/IEEE Frontiers in Education Conference, Boston, MA.

Gillet, D.; Salzmann, C.; Longchamp, R. y Bonvin, D. (1997). Telepresence: An Opportunity to Develop Practical Experimentation in Automatic Control Education. European Control Conference, Bruselas, Belgica.

Hahn, H.H. y Spong, M.W. (2000). Remote Laboratories for Control Education. Proceedings of the 39th IEEE Conference on Decision and Control, Sydney Australia.

Henry, J. (1999). Using the World Wide Web for Teaching Control Systems Design. Proceedings of the 1999 IEEE International Symposium on Computer Aided Control System Design, Koala Coast-Islands of Hawai, Hawai, pp.291-296.

Jing Z; Jianping C.; C. C. Ko; Ben M. y Chen, Ge, (2001). A Web-Based Laboratory on Control of a TwoDegree-of- Freedom Helicopter. Conference on Decision and Control, Orlando, Florida.

Ko, C.C.; Chen, B.M.; Chen, J.; Zhuang, Y. y Tan, K.C. (2001). Development of a Web-Based Laboratory for Control Experiments on a Coupled Tank Apparatus. IEEE Transactions On Education, pp.7686.

Leiner, R. (2002). Tele-Experiments Via Internet- A New Approach For Distance Education. 12th Mediterranean Electrotechnical Conference, Cairo, Egipto.

Messom, C. y Craig, R. (2002). Web Based Laboratory for Controlling Real Robot Systems. Proceedings of The Biannual Conference of the Distance Education Association of New Zealand, Wellington, Nueva Zelandia.

Piguet, Y. y Guillet, D. (1999). Java Based Remote Experimentation for Control Algorithms Prototyping. American Control Conference, San Diego, California.

Poindexter, S.E. y Heck, B.S., 1999, "Using the web in your courses: What can you do? What should you do?". IEEE Control Systems Magazine pp. 83-92. 
Puerto, R.; Reinoso, O.; Ñeco, R.; García, N. y Jiménez, L.M. (2001). Remote Lab for Control Applications Using Matlab. Proceedings on Internet Based Control Education, Madrid, España.

Sebastián, D. (2002). Control learning: present and future. 15th IFAC World Congress, Barcelona, España.

Shor, M.H. (2000). Remote-Access Engineering Educational Laboratories: Who, What, When, Where, Why, and How? Proceedings of the American Control Conference, Chicago, Illinois, pp.2949-2950.

Slotine, J.J. y W., Li, 1991, "Applied nonlinear control", Prentice Hall. 